\title{
New Flutter Analysis Technique for Time-Domain Computational Aeroelasticity
}

\author{
Chan-gi Pak ${ }^{1}$ and Shun-fat Lung ${ }^{2}$ \\ NASA Armstrong Flight Research Center, Edwards, California 93523-0273
}

\begin{abstract}
A new time-domain approach for computing flutter speed is presented. Based on the timehistory result of aeroelastic simulation, the unknown unsteady aerodynamics model is estimated using a system identification technique. The full aeroelastic model is generated via coupling the estimated unsteady aerodynamic model with the known linear structure model. The critical dynamic pressure is computed and used in the subsequent simulation until the convergence of the critical dynamic pressure is achieved. The proposed method is applied to a benchmark cantilevered rectangular wing.
\end{abstract}

\section{Nomenclature}

$\mathbf{A}, \mathbf{B}=$ state-space matrices for structural system

$\mathbf{A}_{\mathrm{a}}, \mathbf{B}_{\mathrm{a}}, \mathbf{C}_{\mathrm{a}}, \mathbf{D}_{\mathrm{a}}=$ state-space matrices for aerodynamics system

$\mathrm{C}=$ damping matrix

$\mathrm{CFD}=$ computational fluid dynamics

$\mathrm{cg} \quad=$ center of gravity

DOF $=$ degrees of freedom

$\mathrm{d} t=$ time step

$\mathrm{FE} \quad=$ finite element

FEM $=$ finite element method

$\mathrm{f} \quad=$ frequency, $\mathrm{Hz}$

$\mathrm{g} \quad=$ structural damping

$\mathbf{K}=$ stiffness matrix

$\mathbf{M} \quad=$ mass matrix

MIMO = multi-input multi-output

$\mathbf{N}=$ orthonormalized aerodynamic force vector

$\mathrm{n}=$ number of mode

$\mathrm{n} 4 \mathrm{sid}=\mathrm{a} \mathrm{MATLAB}^{\circledR}$ (The MathWorks, Inc., Natick, Massachussetts) subspace system identification algorithm

PSD $\quad=$ power spectral density

Q $\quad=$ generalized aerodynamic force vector

$\mathbf{q} \quad=$ generalized displacement vector

$\mathrm{q}_{\mathrm{D}} \quad=$ dynamic pressure

$r, s=$ real and imaginary part of the eigenvalue

SOCIT $=$ System/Observer/Controller Identification Toolbox

$\mathrm{s} \quad=$ Laplace operator

$\mathbf{X}=$ state vector

$\boldsymbol{\beta}=$ damping in the continuous time domain

$\Gamma \quad=$ time integration of a state transition matrix

$\eta \quad=$ orthonormalized displacement vector

$\Theta \quad=$ Г B

$\Lambda \quad=$ eigenvalues of the aeroelastic system

$\sigma \quad=$ state-difference variable

$\tau=$ state-difference variable

\footnotetext{
${ }^{1}$ Senior Aerospace Engineer, Aerostructures Branch, P.O. Box 273 / MS 48201A, Senior AIAA Member.

${ }^{2}$ Contractor, Aerostructures Branch, P.O. Box 273 / MS 4840D, AIAA Member.
} 


$\begin{array}{ll}\Phi & =\text { eigenmatrix (mode shapes) } \\ \boldsymbol{\Psi} & =\text { state transition matrix } \\ \omega & =\text { undamp natural frequency } \\ \omega_{\mathbf{d}} & =\text { damp natural frequency }\end{array}$

\section{Introduction}

$\mathrm{T}$ o reduce the time and expense spent on the design and analysis of an aircraft, it has been desirable to decrease the amount of wind-tunnel flutter test by utilizing numerical simulations. Various computational fluid dynamics (CFD) codes such as CAP-TSDv ${ }^{1}, \mathrm{CFL}_{3} \mathrm{D}^{2}$, and FUN3D ${ }^{3}$ have been developed for this purpose.

The classical method of determining the flutter speed from CFD results is a tedius process. The process requires the user to run numerous time-consuming CFD simulations while varying the dynamic pressure until the critical dynamic pressure is approximately determined by using a trial-and-error process. Varius efforts to identify the flutter boundaries directly from the aeroelastic response ${ }^{4,5}$ have been made based on system identification technique. The least-squares curve fitting method described in Ref. 4 required a large number of sampling points in order to produce reasonable results, and this method assumes that an aeroelastic system was linear within the time range of curve fitting. This fitting technique is basically based on a nonlinear least-squares method because of the sine, cosine, and exponential functions to be used. This approach worked well only with good initial system damping and frequency values. On the other hand, the time-domain flutter analysis technique described in Ref. 5 was numerically efficient and piecewise linear assumptions were used. Both structural and unsteady aerodynamic systems were assumed to be unknown systems, and therefore a system identification technique based on a single-input single-output parameter estimation together with an Autoregressive Moving Average (ARMA) model was applied to the time histories of aeroelastic responses. System damping and frequency values can be obtained from CFD results, however, this approach can not provide a guideline about the dynamic pressure for future CFD computations.

This paper presents a flutter analysis technique for time-domain unsteady computational aeroelasticity. The technique uses an iterative approach to determine the critical dynamic pressure for a given Mach number. The model of the full aeroelastic system is created by coupling the estimated unsteady aerodynamic model with the known linear structural dynamic model. The multi-input-multi-output (MIMO) model of the nonlinear aerodynamics is created utilizing system identification techniques. Updated unsteady aerodynamic models are estimated at every iteration. The critical dynamic pressure is determined based on the full aeroelastic model and is used in the subsequent CFD simulation until convergence is achieved. The critical dynamic pressure is computed based on the $q_{D}-\mathrm{g}$ (dynamic pressure versus damping) and $q_{D}-f$ (dynamic pressure versus frequency) plots. The major benefit of using the current approach is that the classical $q_{D}-\mathrm{g}$ and $q_{D}-f$ plots for the frequency-domain flutter analysis technique can be used with time-domain aeroelasticity data. The critical dynamic pressure value computed in the current iteration can be a good candidate dynamic pressure for the next CFD simulation. A cantilevered rectangular wing ${ }^{6}$ is used as the test case for the method presented.

\section{Method and Tools}

The following section describes the method and tools used in the process of applying the system identification technique and computing flutter speeds. The structural system is linear and the structural dynamic model is known.

\section{A. Interpolation between Structural Dynamic and Unsteady Aerodynamic Models}

The computational aeroelastic code utilized in this study is the CFL3D v.6 code, developed at the NASA Langley Research Center (LaRC) (Hampton, Virginia). The aeroelastic capability, including the automated grid deformation scheme of CFL3D v.6, utilizes either single-grid or point-to-point matching multiblock structured grid. The structural dynamic model and the modal analysis results are considered known prior to CFD simulation. The computational grids typically do not match due to resolution difference between the structural dynamic and unsteady aerodynamic models. The structural mode shapes on the surface grids of the CFD model are computed using the following simple procedure instead of using the Harder and Desmarais surface-spline technique ${ }^{7}$ which has the well-known numerical problem associated with the selection of the proper splining points.

1) Include surface grids of the CFD model in the structural finite element (FE) model. Connect these surface CFD grids to the nearest structural finite element method (FEM) grids (four for quadrilateral and three for triangular elements) using an interpolation constraint element ${ }^{8}$ (RBE3 element in MSC/Nastran ${ }^{\mathrm{TM}}$ (MSC Software Corporation, Newport Beach, California) ${ }^{9}$ terminology) as shown in Fig. 1. In this figure, motion of CFD grids are dependent on the FEM grids. Therefore, neither the stiffness nor the mass properties of the FE model are affected by the CFD grids. 
2) Perform modal analysis. Structural mode shapes are displayed using deflections at FEM grids. On the other hand, mode shapes based on CFD grids are the structural mode shapes on the aerodynamic model to be used for CFD computations.

\section{B. Generation of Estimated Unsteady Aerodynamic Model}

The unknown unsteady aerodynamic model is created using a system identification technique. The process is:

1) Perfom static CFD computation with rigid structure.

2) Create surface CFD grids, and connect these grids to the FEM grids using interpolation elements. Perform modal analysis using the structural FE model; then mode shapes on the surface CFD grids will be obtained.

3) Perform steady CFD computation with flexible structure (mode shapes on the surface CFD grids).

4) Perform unsteady CFD computation with flexible structure using initial velocity conditions.

5) Obatin time histories of the orthonormalized aerodynamic force and displacement from the unsteady CFD run.

6) Generate the MIMO state-space model using a system identification tool such as System/Observer/Controller Identification Toolbox (SOCIT) ${ }^{10}$ or n4sid (The MathWorks, Inc., Natick, Massachussetts) ${ }^{11}$.

\section{Proposed CFD-based Aeroelastic Analysis Technique}

The proposed time-domain technique utilizes the coupled model of the estimated unsteady aerodynamic model and the linear structure model to compute the critical dynamic pressure. State-space difference and measurement equations for an unknown aerodynamic system in the discrete time-domain can be estimated using a system identification tool and be written as shown in Eq. (1)

and Eq. (2)

$$
\boldsymbol{X}_{k+1}=\mathbf{A}_{a} \boldsymbol{X}_{k}+\mathbf{B}_{a} \boldsymbol{\eta}_{k}
$$

$$
\boldsymbol{N}_{k}=q_{D} \mathbf{C}_{a} \boldsymbol{X}_{k}+q_{D} \mathbf{D}_{a} \boldsymbol{\eta}_{k}
$$

where, $\boldsymbol{\eta}_{k}$ and $\boldsymbol{N}_{k}$ are orthonormalized displacement and aerodynamic force vectors at discrete time $k$, respectively.

Consider the following structural dynamic differential equations of motion in matrix form, as shown in Eq. (3).

$$
\mathbf{M} \ddot{\boldsymbol{q}}+\mathbf{C} \dot{\boldsymbol{q}}+\mathbf{K q}=\boldsymbol{Q}
$$

The generalized displacement vector $\boldsymbol{q}$ can be defined as shown in Eq. (4)

$$
\boldsymbol{q} \equiv \boldsymbol{\Phi} \boldsymbol{\eta}
$$

where, $\boldsymbol{\Phi}$ and $\boldsymbol{\eta}$ are an eigenmatrix and a orthonormalized displacement vector, respectively. Substituting Eq. (4) into Eq.(3) gives Eq. (5).

$$
\mathbf{M \Phi} \ddot{\eta}+\mathbf{C} \boldsymbol{\dot { \eta }}+\mathbf{K \Phi} \boldsymbol{\eta}=Q
$$

Pre-multiplying $\boldsymbol{\Phi}^{T}$ to Eq.(5) gives Eq. (6)

$$
\ddot{\eta}+2 \zeta \omega \dot{\eta}+\omega^{2} \eta=N
$$

where $\boldsymbol{\Phi}^{T} \mathbf{M} \boldsymbol{\Phi}=\boldsymbol{I}, \boldsymbol{\Phi}^{T} \mathbf{K} \boldsymbol{\Phi}=\boldsymbol{\omega}^{2}, \boldsymbol{\Phi}^{T} \mathbf{C} \boldsymbol{\Phi}=2 \zeta \boldsymbol{\omega}$, and $\boldsymbol{N}=\boldsymbol{\Phi}^{T} \boldsymbol{Q}$.

Then Eq.(6) can be converted to the state-differential equation shown as Eq. (7)

$$
\left\{\begin{array}{l}
\dot{\boldsymbol{\eta}} \\
\ddot{\boldsymbol{\eta}}
\end{array}\right\}=A\left\{\begin{array}{c}
\boldsymbol{\eta} \\
\dot{\boldsymbol{\eta}}
\end{array}\right\}+B N
$$

where $\boldsymbol{A}=\left[\begin{array}{cc}\mathbf{0} & \boldsymbol{I} \\ -\boldsymbol{\omega}^{2} & -\mathbf{2 \zeta} \boldsymbol{\omega}\end{array}\right]$ and $\boldsymbol{B}=\left[\begin{array}{l}\mathbf{0} \\ \boldsymbol{I}\end{array}\right]$

The state-differential equation, Eq.(7), can be changed to the state-difference equation shown as Eq. (8). ${ }^{12}$ 


$$
\left\{\begin{array}{l}
\boldsymbol{\eta} \\
\dot{\boldsymbol{\eta}}
\end{array}\right\}_{k+1}=\boldsymbol{\Psi}\left\{\begin{array}{l}
\boldsymbol{\eta} \\
\dot{\boldsymbol{\eta}}
\end{array}\right\}_{k}+\Theta\left(\frac{\boldsymbol{N}_{k}+N_{k+1}}{2}\right)
$$

Detailed derivation of Eq.(8) is given in the appendix. Rewrite Eq.(8) in partitioned form, resulting in Eq. (9).

$$
\left\{\begin{array}{l}
\boldsymbol{\eta} \\
\dot{\boldsymbol{\eta}}
\end{array}\right\}_{k+1}=\left[\begin{array}{ll}
\boldsymbol{\Psi}_{11} & \boldsymbol{\Psi}_{12} \\
\boldsymbol{\Psi}_{21} & \Psi_{22}
\end{array}\right]\left\{\begin{array}{l}
\boldsymbol{\eta} \\
\dot{\eta}
\end{array}\right\}_{k}+\left[\begin{array}{l}
\boldsymbol{\Theta}_{1} \\
\boldsymbol{\Theta}_{2}
\end{array}\right]\left(\frac{N_{k}+N_{k+1}}{2}\right)
$$

Substituting Eq.(2) into Eq.(9) gives Eq. (10),

$$
\left\{\begin{array}{l}
\boldsymbol{\eta}_{\boldsymbol{\eta}} \\
\dot{\boldsymbol{\eta}}
\end{array}\right\}_{k+1}=\left[\begin{array}{ll}
\boldsymbol{\Psi}_{11} & \boldsymbol{\Psi}_{\mathbf{1 2}} \\
\boldsymbol{\Psi}_{\mathbf{2 1}} & \boldsymbol{\Psi}_{22}
\end{array}\right]\left\{\begin{array}{l}
\boldsymbol{\eta}_{\boldsymbol{\eta}} \\
\dot{\eta}
\end{array}\right\}+\left[\begin{array}{l}
\boldsymbol{\Theta}_{\mathbf{1}} \\
\boldsymbol{\Theta}_{2}
\end{array}\right]\left(\frac{q_{D}}{2} \mathbf{C}_{a} \boldsymbol{X}_{k}+\frac{q_{D}}{2} \mathbf{D}_{a} \boldsymbol{\eta}_{k}+\frac{q_{D}}{2} \mathbf{C}_{a} \boldsymbol{X}_{k+1}+\frac{q_{D}}{2} \mathbf{D}_{a} \boldsymbol{\eta}_{k+1}\right)
$$

that is, Eqs. (11) and (12).

$$
\begin{aligned}
& \boldsymbol{\eta}_{k+1}=\boldsymbol{\Psi}_{\mathbf{1 1}} \boldsymbol{\eta}_{k}+\boldsymbol{\Psi}_{\mathbf{1 2}} \dot{\boldsymbol{\eta}}_{k}+\frac{q_{D}}{2} \boldsymbol{\Theta}_{\mathbf{1}} \mathbf{C}_{a} \boldsymbol{X}_{k}+\frac{q_{D}}{2} \boldsymbol{\Theta}_{\mathbf{1}} \mathbf{D}_{a} \boldsymbol{\eta}_{k}+\frac{q_{D}}{2} \boldsymbol{\Theta}_{\mathbf{1}} \mathbf{C}_{a} \boldsymbol{X}_{k+1}+\frac{q_{D}}{2} \boldsymbol{\Theta}_{\mathbf{1}} \mathbf{D}_{a} \boldsymbol{\eta}_{k+1} \\
& \dot{\boldsymbol{\eta}}_{k+1}=\boldsymbol{\Psi}_{\mathbf{2 1}} \boldsymbol{\eta}_{k}+\boldsymbol{\Psi}_{\mathbf{2 2}} \dot{\boldsymbol{\eta}}_{k}+\frac{q_{D}}{2} \boldsymbol{\Theta}_{\mathbf{2}} \mathbf{C}_{a} \boldsymbol{X}_{k}+\frac{q_{D}}{2} \boldsymbol{\Theta}_{\mathbf{2}} \mathbf{D}_{a} \boldsymbol{\eta}_{k}+\frac{q_{D}}{2} \boldsymbol{\Theta}_{\mathbf{2}} \mathbf{C}_{a} \boldsymbol{X}_{k+1}+\frac{q_{D}}{2} \boldsymbol{\Theta}_{\mathbf{2}} \mathbf{D}_{a} \boldsymbol{\eta}_{k+1}
\end{aligned}
$$

Rearranging Eqs.(11) and (12) gives Eqs. (13) and (14).

$$
\begin{aligned}
& \left(\boldsymbol{I}-\frac{q_{D}}{2} \boldsymbol{\Theta}_{\mathbf{1}} \mathbf{D}_{a}\right) \boldsymbol{\eta}_{k+1}-\frac{q_{D}}{2} \boldsymbol{\Theta}_{\mathbf{1}} \mathbf{C}_{a} \boldsymbol{X}_{k+1}=\boldsymbol{\Psi}_{\mathbf{1 1}} \boldsymbol{\eta}_{k}+\boldsymbol{\Psi}_{\mathbf{1 2}} \dot{\boldsymbol{\eta}}_{k}+\frac{q_{D}}{2} \boldsymbol{\Theta}_{\mathbf{1}} \mathbf{C}_{a} \boldsymbol{X}_{k}+\frac{q_{D}}{2} \boldsymbol{\Theta}_{\mathbf{1}} \mathbf{D}_{a} \boldsymbol{\eta}_{k} \\
& -\frac{q_{D}}{2} \boldsymbol{\Theta}_{\mathbf{2}} \mathbf{D}_{a} \boldsymbol{\eta}_{k+1}+\dot{\boldsymbol{\eta}}_{k+1}-\frac{q_{D}}{2} \boldsymbol{\Theta}_{\mathbf{2}} \mathbf{C}_{a} \boldsymbol{X}_{k+1}=\boldsymbol{\Psi}_{\mathbf{2 1}} \boldsymbol{\eta}_{k}+\boldsymbol{\Psi}_{\mathbf{2} 2} \dot{\boldsymbol{\eta}}_{k}+\frac{q_{D}}{2} \boldsymbol{\Theta}_{\mathbf{2}} \mathbf{C}_{a} \boldsymbol{X}_{k}+\frac{q_{D}}{2} \boldsymbol{\Theta}_{\mathbf{2}} \mathbf{D}_{a} \boldsymbol{\eta}_{k}
\end{aligned}
$$

Define a state vector, $\widetilde{\boldsymbol{X}}$, at discrete time $k$ as shown in Eq. (15),

$$
\widetilde{\boldsymbol{X}}_{\boldsymbol{k}} \equiv\left\{\begin{array}{l}
\boldsymbol{\eta} \\
\dot{\boldsymbol{\eta}} \\
\boldsymbol{X}
\end{array}\right\}_{k}
$$

then from Eqs.(1), (13), and (14), the state difference equation for an aeroelastic system can be obtained, as shown in Eq. (16)

$$
A \widetilde{X}_{k+1}=B \widetilde{X}_{k}
$$

where $\mathbf{A}=\left[\begin{array}{ccc}\boldsymbol{I}-\frac{q_{D}}{2} \boldsymbol{\Theta}_{\mathbf{1}} \mathbf{D}_{a} & \mathbf{0} & -\frac{q_{D}}{2} \boldsymbol{\Theta}_{\mathbf{1}} \mathbf{C}_{a} \\ -\frac{q_{D}}{2} \boldsymbol{\Theta}_{\mathbf{2}} \mathbf{D}_{a} & \boldsymbol{I} & -\frac{q_{D}}{2} \boldsymbol{\Theta}_{\mathbf{2}} \mathbf{C}_{a} \\ \mathbf{0} & \mathbf{0} & \mathbf{I}\end{array}\right] \quad$ and $\quad \boldsymbol{B}=\left[\begin{array}{ccc}\boldsymbol{\Psi}_{\mathbf{1 1}}+\frac{q_{D}}{2} \boldsymbol{\Theta}_{\mathbf{1}} \mathbf{D}_{a} & \boldsymbol{\Psi}_{\mathbf{1 2}} & \frac{q_{D}}{2} \boldsymbol{\Theta}_{\mathbf{1}} \mathbf{C}_{a} \\ \boldsymbol{\Psi}_{\mathbf{2 1}}+\frac{q_{D}}{2} \boldsymbol{\Theta}_{\mathbf{2}} \mathbf{D}_{a} & \boldsymbol{\Psi}_{\mathbf{2 2}} & \frac{q_{D}}{2} \boldsymbol{\Theta}_{\mathbf{2}} \mathbf{C}_{a} \\ \mathbf{B}_{a} & \mathbf{0} & \mathbf{A}_{a}\end{array}\right]$

\section{Summary of Procedure}

The procedure for the new flutter speed computation using CFD-based aeroelastic analysis combined with the system identification technique is summarized as shown in Fig. 2. This process starts with the FE model and computations of natural frequencies and mode shapes. The CFD-based aeroelastic analysis with an arbitrary Mach number and dynamic pressure is then carried out in Step 1. Time-history data of the orthonormalized aerodynamic forces obtained from Step 1 are then non-dimensionalized by $q_{D}$ in Step 2 . With each element of the orthonormalized displacement vector from Step 1 as multi-input and each element of the non-dimensional orthonormalized aerodynamic force vectors, $\boldsymbol{N}_{k} / \boldsymbol{q}_{\boldsymbol{D}}$, from Step 2 as multi-output, the state difference equation for an aerodynamic system can be generated using the system identification tool in Step 3. Step 4 combines the known structural dynamic model with the estimated aerodynamic model to form the state difference equation for an aeroelastic system. By solving the state difference equation with a different dynamic pressure, one can determine the critical dynamic pressure using the $q_{D}-\mathrm{g}$ and $q_{D}-f$ plots, which process is similar to 
classical flutter analysis with lifting surface theory. Repeat Step 1 through Step 4 with the updated dynamic pressure until it is converged. A reasonable starting dynamic pressure can be obtained from a linear panel method or test data.

The governing equation in Eq.(16) is based on discrete time $k$, and therefore aeroelastic system damping and frequencies from Eq.(16) should be converted to continuous time damping and frequencies. The eigenvalues $\boldsymbol{\Lambda}_{\boldsymbol{j}}$ obtained from Eq.(16) can be written as shown in Eq. (17)

$$
\boldsymbol{\Lambda}_{\boldsymbol{j}}=\boldsymbol{r}_{\boldsymbol{j}} \pm \boldsymbol{i} \boldsymbol{s}_{\boldsymbol{j}} \quad j=1,2, \ldots, n
$$

where, $\boldsymbol{r}_{\boldsymbol{j}}$ and $\boldsymbol{s}_{\boldsymbol{j}}$ are the real and imaginary part of the eigenvalues obtained from Eq.(16). Aeroelastic damping and damped frequencies, $\boldsymbol{\beta}_{\boldsymbol{j}}$ and $\boldsymbol{\omega}_{\boldsymbol{d} \boldsymbol{j}}$, in the continuous time domain are obtained as shown in Eqs. (18) and (19). ${ }^{5}$

$$
\begin{gathered}
\boldsymbol{\beta}_{j}=\frac{1}{2 \Delta T} \log _{e}\left(\boldsymbol{r}_{j}^{2}+\boldsymbol{s}_{j}^{2}\right) \\
\boldsymbol{\omega}_{d j}=\frac{1}{\Delta T} \tan ^{-1} \frac{s_{j}}{r_{j}} .
\end{gathered}
$$

\section{Test Case}

The proposed technique is tested on a cantilevered rectangular wing. This cantilevered rectangular wing with 6-percent circular arc cross sections and an aspect ratio of 5.0 was built and tested at NASA LaRC in $1959^{6}$. The model has a uniform chord length of 4.56 inches, a span length of 11.5 inches, and a 0.065 -inch-thick aluminum insert covered with flexible plastic foam, as shown in Fig. 3. Detailed material properties are shown in Table 1. The shaped lumped weights are used to match the local cross-sectional weight distribution of the plastic foam. Therefore, the small lumped weights are used near the leading- and trailing-edges and the large lumped weights are used near the mid-chord area.

The transformation procedure shown in Figs. 3-5 is for demonstration purposes. Fig. 4 depicts the interpolation (RBE3) elements between FEM and CFD grids used for the cantilevered rectangular wing. The first three mode shapes obtained from MSC/Nastran ${ }^{\mathrm{TM}}$ computation are shown in Fig. 5(a). These structural mode shapes were transfered to the surface CFD grid using interpolation constraint elements using all the FEM grids as master degrees of freedom and all the CFD grids as slave degrees of freedom. Modal analysis used the combined model to obtain the mode shapes on the CFD grid locations. The deflection at the CFD grids were used during CFD simulations. The interpolated mode shapes on the CFD grids are shown in Fig. 5(b).

The CFL3D v.6 code with the Euler option was used for solving the 3-D flows on the structured CFD grid. The computational domain and surface grid for the rectangular wing is a multi-block ( $97 \mathrm{x} 49$ x 41) grid with H-H topology. The top half of the grid symmetry plane (above the airfoil) is shown in Fig. 6. A mirror image of the upper block was used for the lower block.

The aeroelastic computations started from a static aerodynamic computation with rigid structure. Fig. 7(a) shows the local Mach number distributions for the static solution at Mach 0.714. The restarted file from the static solution was then used for the steady-state aeroelastic analysis without any disturbances. After the steady-state aeroelastic analysis had converged, a dynamic aeroelastic analysis was carried out with the initial dynamic pressure $q_{D}=1.0$ psi. The orthonormalized displacement and aerodynamic force vectors from the dynamic aeroelastic analysis were then used as the multi-input and multi-output parameters for the system identification technique to identify the aerodynamic state matrices $\mathbf{A}_{\mathbf{a}}, \mathbf{B}_{\mathbf{a}}, \mathbf{C}_{\mathbf{a}}$, and $\mathbf{D}_{\mathbf{a}}$. The orthonormalized aerodynamic force vectors are normalized by the given dynamic pressure before input to the system identification technique. The coupled aeroelastic state difference matrix in Eq.(16) was solved by the general asymmetric eigenvalue solver with different dynamic pressures. Frequency and damping values were calculated using Eqs. (18) and (19). From the dynamic pressure vs. damping $\left(q_{D}-\mathrm{g}\right)$ plot, the dynamic pressure at the zero damping crossing is obtained. Repeat the dynamic aeroelastic analysis with the critical dynamic pressure obtained from the previous analysis until the critical dynamic pressure converges. Two system identification tools, SOCIT and n4sid, were used to check the robustness of the estimated unsteady aerodynamic models. SOCIT (System/Observer/Controller Identification Toolbox) is a collection of functions that implements a variety of modern system identification techniques. The $\mathrm{n} 4 \mathrm{sid}$ is a built-in subspace system identification technique in MATLAB. The $\left(q_{D}-\mathrm{g}\right)$ and $\left(q_{D}-\mathrm{f}\right)$ plots obtained from each iteration of dynamic pressure using both system identification tools are shown in Figs. 8-10. Estimated unsteady aerodynamic models were slightly different, as shown in these figures, however, there were no difficulties in having converged primary unstable modes with both system identication tools. The SOCIT and n4sid converged at the dynamic pressures of 1.471 and $1.465 \mathrm{psi}$ and the corresponding frequencies of 33.47 and $33.37 \mathrm{~Hz}$, respectively, in three iterations. Both SOCIT and n4sid identify the primary unstable mode correctly. However, often the secondary unstable mode is 
different, as shown in Fig. 10. The time histories of orthonormalized displacement data with stable and unstable dynamic pressures are depicted in Fig. 11.

The flutter boundaries obtained from the wind-tunnel testing ${ }^{6}$ for this cantilevered rectangular wing are summarized in Table 2. The flutter boundaries at a Mach number of 0.714 using the proposed approach are compared with test data in Fig. 12. The computed flutter boundaries at additional Mach numbers, $0.795,0.851,0.913,0.956$, and 1.017 are also shown in Fig. 12. The local Mach number contour from steady CFD computations at corresponding Mach numbers are given in Figs. 7(b) through 7(f). Transonic bucket effect is successfully captured in (Mach $\left.-q_{D}\right)$ curve as shown in Fig. 12(a); however, it failed to obtain the correct flutter frequency in the transonic speed regime, as shown in Fig. 12(b). Time histories and PSDs of the first three orthonormalized displacements at Mach 0.913 and dynamic pressure of 1.33 psi are shown in Fig. 13. In the PSDs, four frequencies, $25 \mathrm{~Hz}, 36 \mathrm{~Hz}, 46 \mathrm{~Hz}$, and $90 \mathrm{~Hz}$, are observed in Figs. 13(b), 13(c), and 13(d). Therefore, the 46-Hz mode was the most dominant mode in the first two orthonormalized displacements, and the system identification code estimated this $46-\mathrm{Hz}$ mode as the first flutter mode. It can be concluded that CFL3D with the Euler option could not provide the correct orthonormalized displacement and force vectors with the first three structural dynamic modes.

\section{Conclusion}

A new time-domain technique for computing flutter speed and frequency based on computational fluid dynamics (CFD) results was presented. The CFL3D v.6 code with the Euler option was used for solving the 3-D flows on the structured grid. The presented method utilizes the system identification technique with orthonormalized displacement and aerodynamic force vectors to create the aerodynamic system model. The full aeroelastic model is created by coupling the estimated aerodynamics model with the known structure dynamic model. The proposed approach is successfully implemented to identify the flutter boundaries of a cantilevered rectangular wing model. Computed flutter speeds and frequencies are in good match with measured quantities, however, the CFL3D code with the Euler option could not provide the correct orthonormalized displacement and force vectors with the first three structural dynamic modes in transonic speed regimes.

In this study, surface grids of the CFD model are included in the structural FE model. These surface CFD grids are connected to the nearest structural finite element method grids using interpolation (RBE3) elements. This proposed fitting technique between structural finite element and CFD models is successfully used in this study instead of using the Harder and Desmarais surface splines.

\section{Appendix}

Integration of Eq.(8) with respect to time yields the state-difference equation shown in Eq. (A.1).

$$
\left\{\begin{array}{l}
\boldsymbol{\eta}\left(\boldsymbol{t}_{\boldsymbol{f}}\right) \\
\dot{\boldsymbol{\eta}}\left(\boldsymbol{t}_{\boldsymbol{f}}\right)
\end{array}\right\}=\mathrm{e}^{A\left(\boldsymbol{t}_{\boldsymbol{f}}-\boldsymbol{t}_{i}\right)}\left\{\begin{array}{l}
\boldsymbol{\eta}\left(\boldsymbol{t}_{\boldsymbol{i}}\right) \\
\dot{\boldsymbol{\eta}}\left(\boldsymbol{t}_{i}\right)
\end{array}\right\}+\int_{\boldsymbol{t}_{\boldsymbol{i}}}^{\boldsymbol{t}_{\boldsymbol{f}}} \mathrm{e}^{A\left(\boldsymbol{t}_{\boldsymbol{f}}-\tau\right)} \boldsymbol{B N}(\tau) d \tau .
$$

Continuous time $t$ in Eq.(A.1) can be changed to discrete time $k$ using the notation shown in Eq. (A.2).

$$
\begin{aligned}
& \boldsymbol{\eta}_{k+1} \equiv \boldsymbol{\eta}\left(\boldsymbol{t}_{\boldsymbol{f}}\right) \\
& \boldsymbol{\eta}_{k} \equiv \boldsymbol{\eta}\left(\boldsymbol{t}_{\boldsymbol{i}}\right) \\
& \boldsymbol{t}_{\boldsymbol{i}} \equiv k \Delta T \\
& \boldsymbol{t}_{\boldsymbol{f}} \equiv(k+1) \Delta T \\
& \Delta T=\boldsymbol{t}_{\boldsymbol{f}}-\boldsymbol{t}_{\boldsymbol{i}} \\
& \boldsymbol{\Psi} \equiv \mathrm{e}^{A \Delta T}
\end{aligned}
$$

Thus, Eq.(A.1) is rewritten as shown in Eq. (A.3).

$$
\left\{\begin{array}{l}
\boldsymbol{\eta} \\
\dot{\boldsymbol{\eta}}
\end{array}\right\}_{k+1}=\boldsymbol{\Psi}\left\{\begin{array}{l}
\boldsymbol{\eta} \\
\dot{\boldsymbol{\eta}}
\end{array}\right\}_{k}+\int_{k \Delta T}^{(k+1) \Delta T} \mathrm{e}^{\boldsymbol{A}\{(k+1) \Delta T-\tau\}} \boldsymbol{B} \boldsymbol{N}(\tau) d \tau
$$

It shoud be noted that Eq.(A.3) is the exact solution of Eq.(7). It is assumed that the orthonormalized aerodynamic force vector $\boldsymbol{N}(t)$ is constant in the small time interval $k \Delta T \leq t \leq(k+1) \Delta T$. In this interval, the orthonormalized aerodynamic 
force vector is assumed to be equal to average value, $\frac{N_{k}+N_{k+1}}{2}$. Therefore, the integration term in Eq.(A.3) can be approximated as shown in Eq. (A.4).

$$
\int_{k \Delta T}^{(k+1) \Delta T} \mathrm{e}^{A\{(k+1) \Delta T-\tau\}} \boldsymbol{B N}(\tau) d \tau \approx \int_{k \Delta T}^{(k+1) \Delta T} \mathrm{e}^{\boldsymbol{A}\{(k+1) \Delta T-\tau\}} d \tau \boldsymbol{B}\left(\frac{\boldsymbol{N}_{k}+\boldsymbol{N}_{k+1}}{2}\right) .
$$

If the variable $\tau$ in Eq.(A.4) is change to $\sigma$ with Eq. (A.5);

$$
\sigma=\tau-k \Delta T .
$$

then the integration term in Eq.(A.4) becomes Eq. (A.6).

$$
\int_{k \Delta T}^{(k+1) \Delta T} \mathrm{e}^{A\{(k+1) \Delta T-\tau\}} d \tau=\int_{0}^{\Delta T} \mathrm{e}^{A(\Delta T-\sigma)} d \sigma \equiv \Gamma .
$$

Therefore, the state-difference equation for the state-differential equation in Eq.(7) can be written as Eq. (A.7).

$$
\left\{\begin{array}{l}
\boldsymbol{\eta} \\
\dot{\eta}
\end{array}\right\}_{k+1}=\Psi\left\{\begin{array}{l}
\boldsymbol{\eta} \\
\dot{\boldsymbol{\eta}}
\end{array}\right\}_{k}+\Gamma \mathrm{\Gamma B}\left(\frac{N_{k}+N_{k+1}}{2}\right)=\Psi\left\{\begin{array}{l}
\boldsymbol{\eta} \\
\dot{\boldsymbol{\eta}}
\end{array}\right\}_{k}+\Theta\left(\frac{N_{k}+N_{k+1}}{2}\right) .
$$

\section{References}

${ }^{1}$ Edwards, J. W., "Transonic Shock Oscillations Calculated with a New Interactive Boundary Layer Coupling Method," AIAA-93-0777.

${ }^{2}$ Krist, S. L., Biedron, R. T., and Rumsey, C. L., “CFL3D User's Manual (Version 5.0),” NASA/TM-1998-208444.

${ }^{3}$ Biedron, R. T., Vatsa, V. N., and Atkins, H. L., "Simulation of Unsteady Flows Using an Unstructured Navier-Stokes Solver on Moving and Stationary Grids," AIAA-2005-5093.

${ }^{4}$ Bennett, R. M., and Desmarais, R. N., "Curve Fitting of Aeroelastic Transient Response Data with Exponential Functions,” NASA-SP-415, pp. 43-58, 1975.

${ }^{5}$ Pak, C.-G., and Friedmann, P. P., "New Time Domain Technique for Flutter Boundary Identification," AIAA-92-2102, AIAA Dynamics Specialist Conference, Washington, D.C., 1992.

${ }^{6}$ Doggett, R. V. Jr., Rainey, A. G., and Morgan, H. G., "An Experimental Investigation of Aerodynamic Effects of Airfoil Thickness on Transonic Flutter Characteristics," NASA-TM X-79, 1959.

${ }^{7}$ Harder, R. L., and Desmarais, R. N., "Interpolation Using Surface Splines," AIAA Journal of Aircraft, Vol. 9, No.2, 1972, pp. 189-191.

${ }^{8}$ Pak, C.-g., "Unsteady Aerodynamic Force Sensing from Measured Strain," Proceedings of $30^{\text {th }}$ Congress of the International Council of the Aeronautical Sciences, Daejeon, Korea, September 25-30, 2016.

${ }^{9}$ MSC Nastran, MSC Software Corporation, http://www.mscsoftware.com/product/msc-nastran, accessed October 26, 2016.

${ }^{10} J u a n g$, J-N, Horta, L., and Phan, M., "System/Observer/Controller Identification Toolbox," NASA TM-107566, 1992.

${ }^{11}$ MATLAB ${ }^{\circledR}$ Desktop Tools and Development Environment, The MathWorks, Inc., Natick, Massachusetts, 2016.

${ }^{12}$ Edwards, J. W., Bennett, R. M., Whitlow, W. Jr., and Seidel, D. A., "Time-Marching Transonic Flutter Solutions Including Angle-of-Attack. Effects," AIAA Journal of Aircraft, Vol. 20, No. 11, pp. 899-906. 


\section{Tables}

Table 1. Detailed material properties of the cantilevered rectangular wing.

\begin{tabular}{|l|c|}
\hline Properties & Number \\
\hline Young's modulus, E, of aluminum insert & $9207766 \mathrm{psi}$ \\
\hline Shear modulus, G, of aluminum insert & $3836570 \mathrm{psi}$ \\
\hline Density of aluminum insert & $0.1 \mathrm{lb} / \mathrm{in}^{\wedge} 3$ \\
\hline Total weight & $0.3806 \mathrm{lb}$ \\
\hline Xcg & $2.28 \mathrm{inch}$ \\
\hline Ycg & $5.75 \mathrm{inch}$ \\
\hline Thickness of aluminum insert & 0.065 inch \\
\hline
\end{tabular}

Table 2. Flutter boundary of the cantilevered rectangular wing.

\begin{tabular}{|l|c|c|}
\hline Mach number & Dynamic pressure, psi & Frequency, Hz \\
\hline 0.714 & 1.450377 & 33.80000 \\
\hline 0.795 & 1.261161 & 31.40127 \\
\hline 0.814 & 1.310468 & 31.59226 \\
\hline 0.836 & 1.198658 & 30.49409 \\
\hline 0.851 & 1.231993 & 29.39592 \\
\hline 0.905 & 1.191714 & 24.70085 \\
\hline 0.907 & 1.236854 & 25.19423 \\
\hline 0.913 & 1.122267 & 24.00057 \\
\hline 0.923 & 1.174352 & 28.20226 \\
\hline 0.927 & 1.133378 & 24.60535 \\
\hline 0.956 & 1.454224 & 30.00071 \\
\hline 1.017 & 1.732707 & 33.6931 \\
\hline
\end{tabular}




\section{Figures}

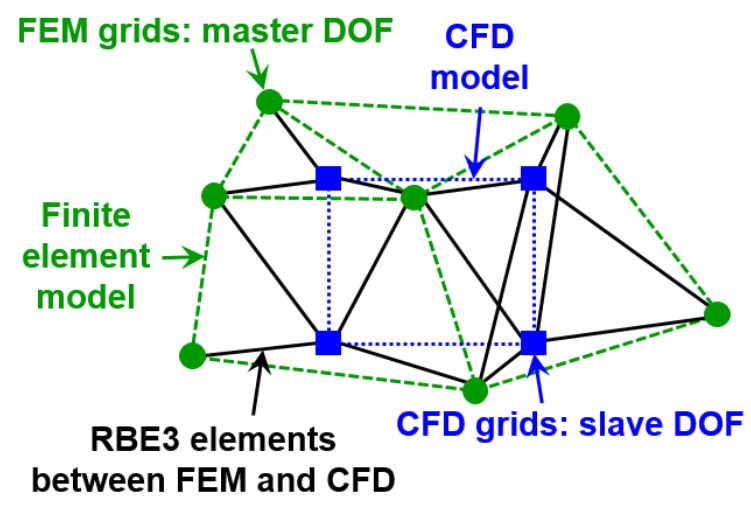

Figure 1. Definition of the FEM and CFD grids used in this study.

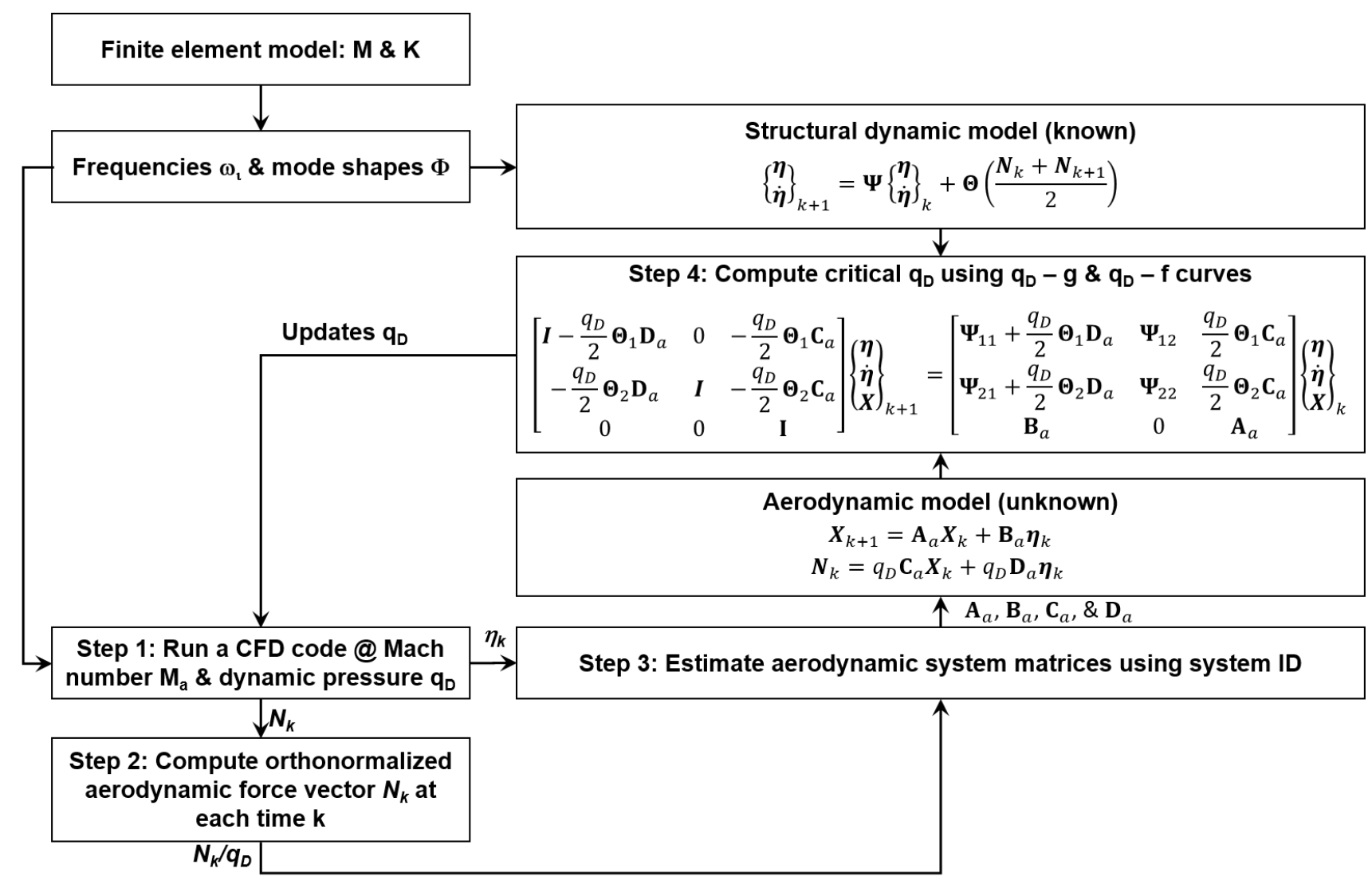

Figure 2. Flow chart showing time-domain CFD-based flutter speed computation. 


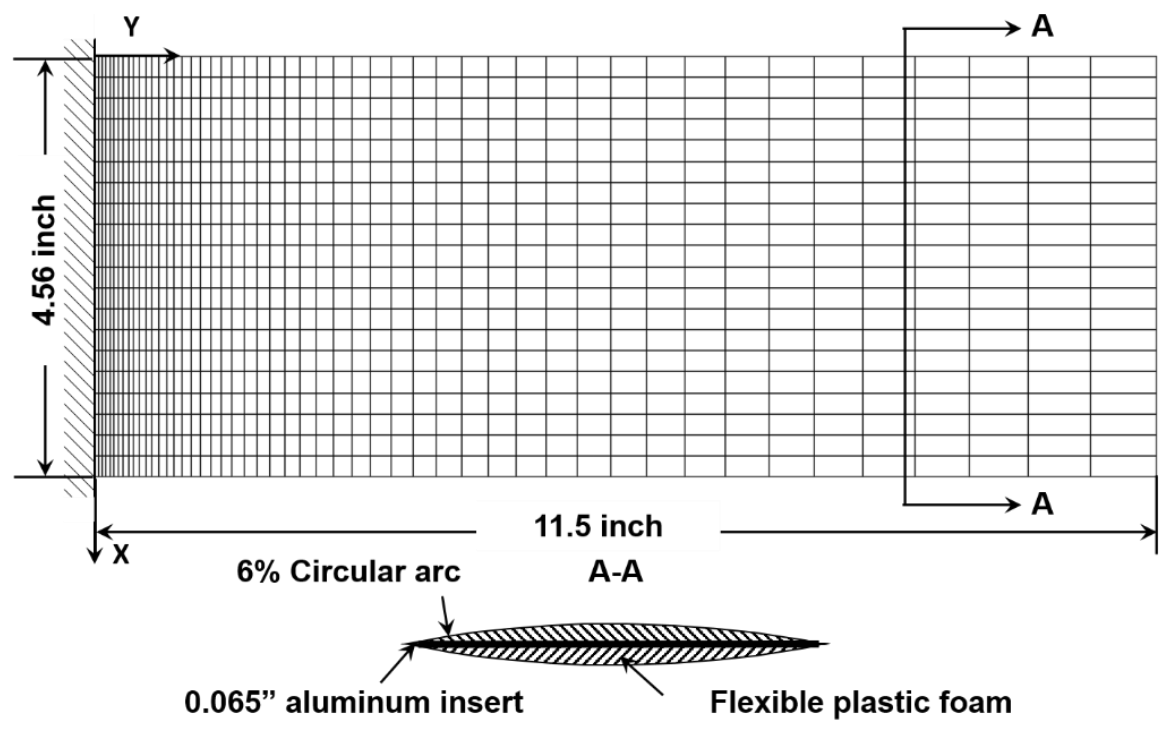

Figure 3. Structural model of the cantilevered rectangular wing.

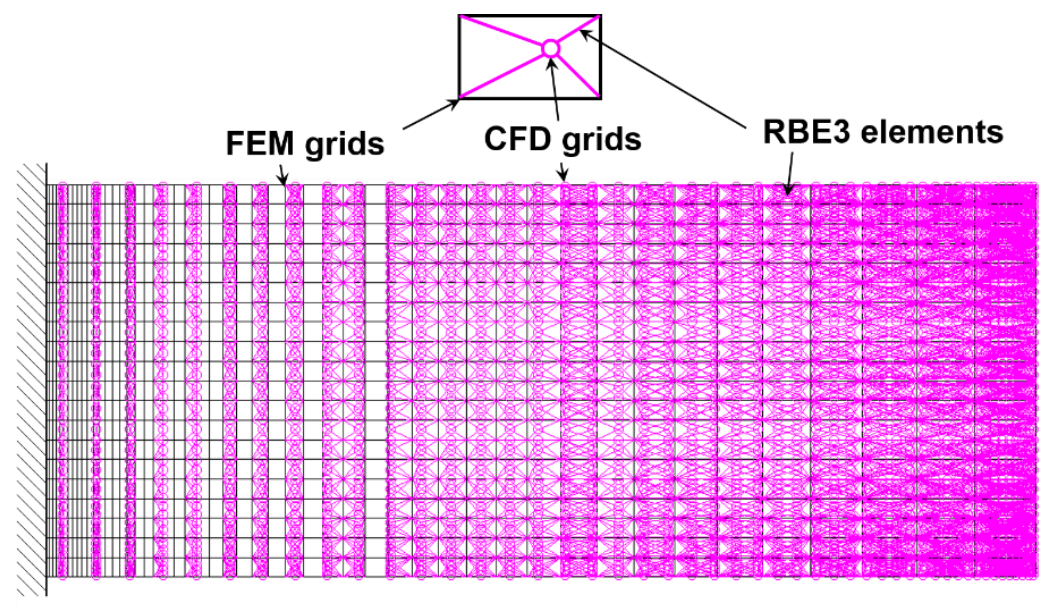

Figure 4. FEM and CFD grids connection using RBE3 elements. 


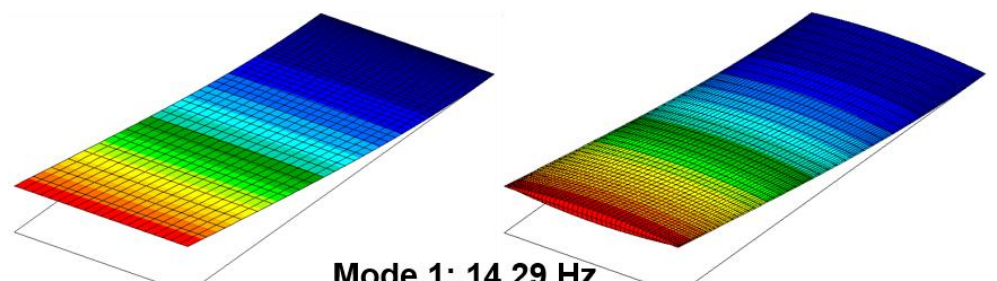

Mode 1: $14.29 \mathrm{~Hz}$
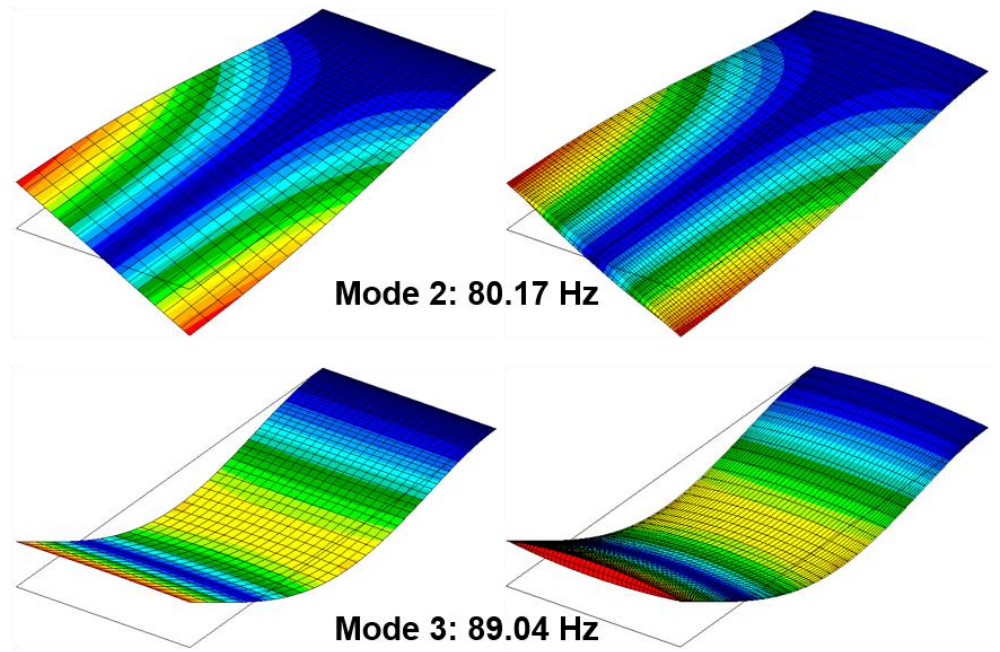

(a) FEM grids

(b) CFD grids

Figure 5. Mode shapes of the cantilevered rectangular wing on structural and aerodynamic models.

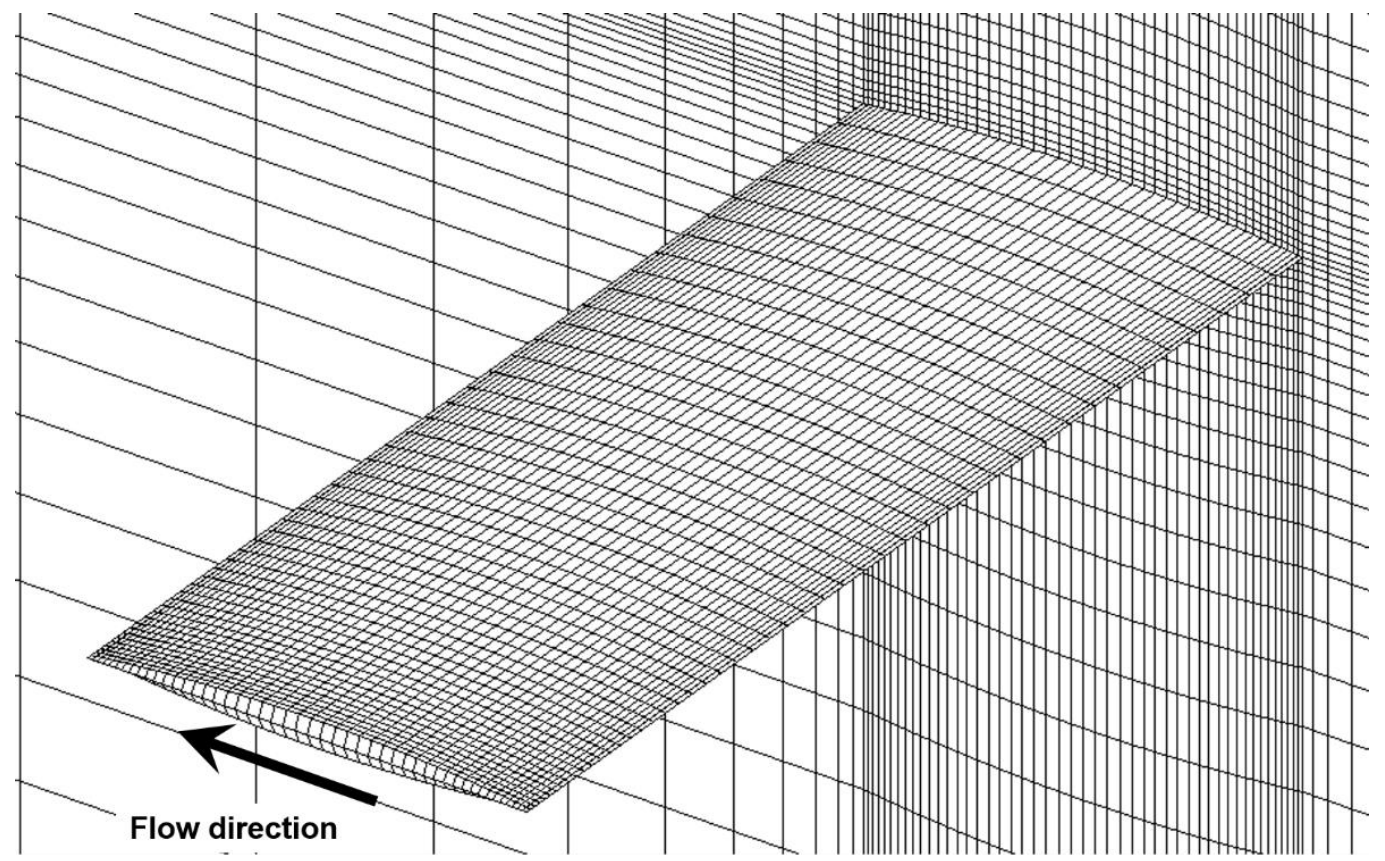

Figure 6. A CFD grid for CFL3D computations based on an Euler grid. 


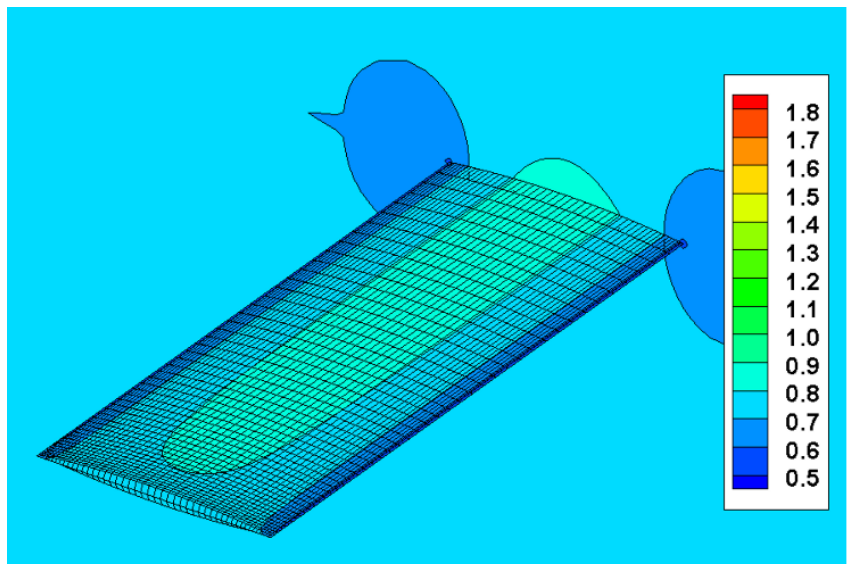

(a) Mach $=0.714$

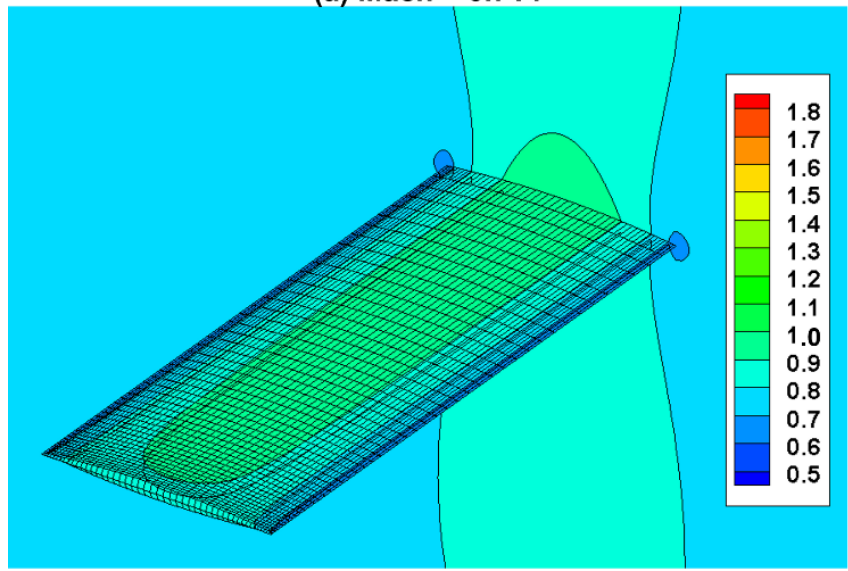

(b) Mach $=0.795$

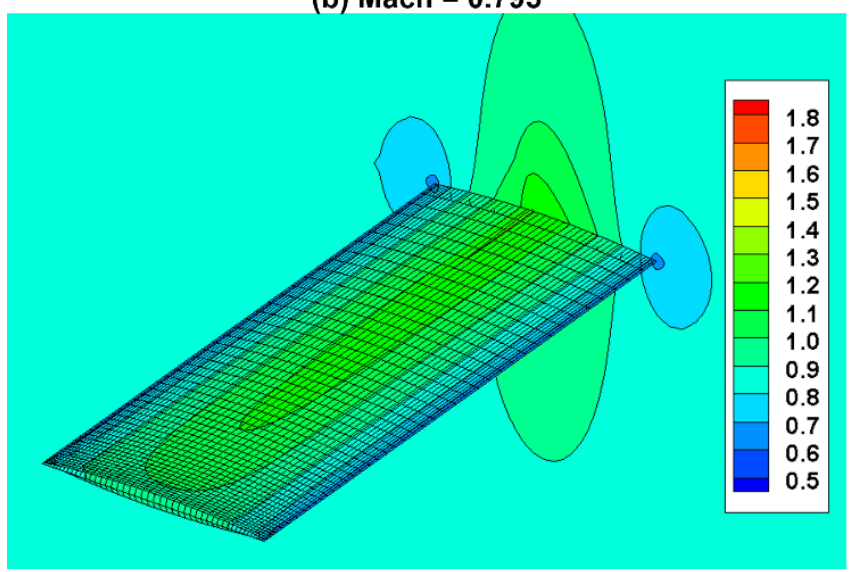

(c) Mach $=0.851$

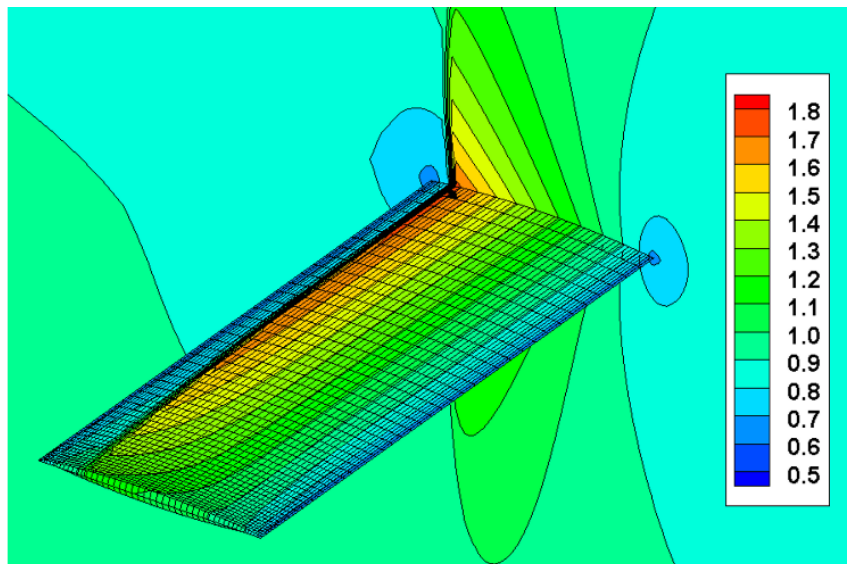

(d) Mach $=0.913$

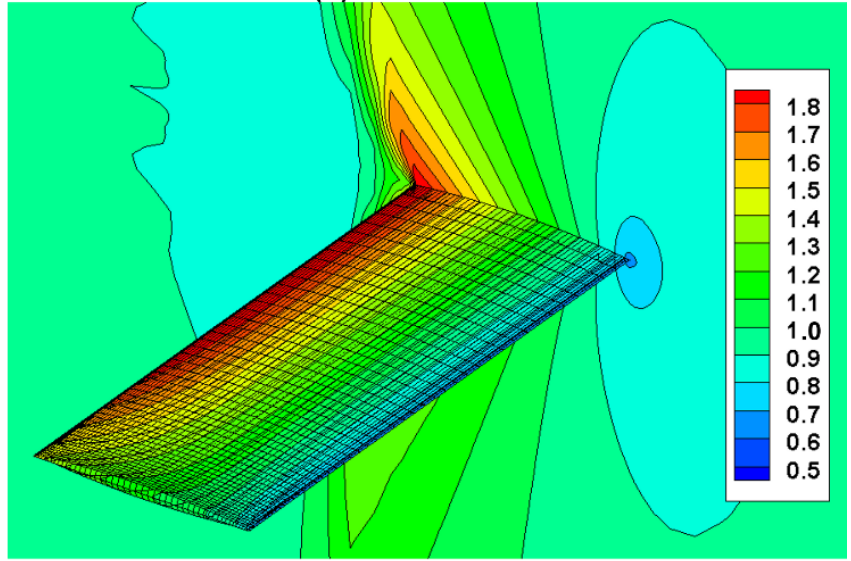

(e) Mach $=0.956$

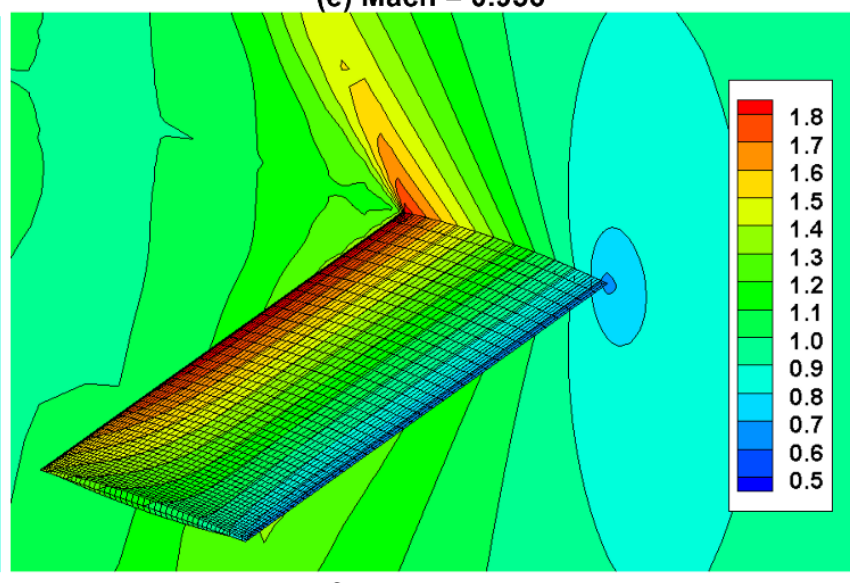

(f) $\operatorname{Mach}=1.017$

Figure 7. Local Mach number contour from steady CFD computations. 

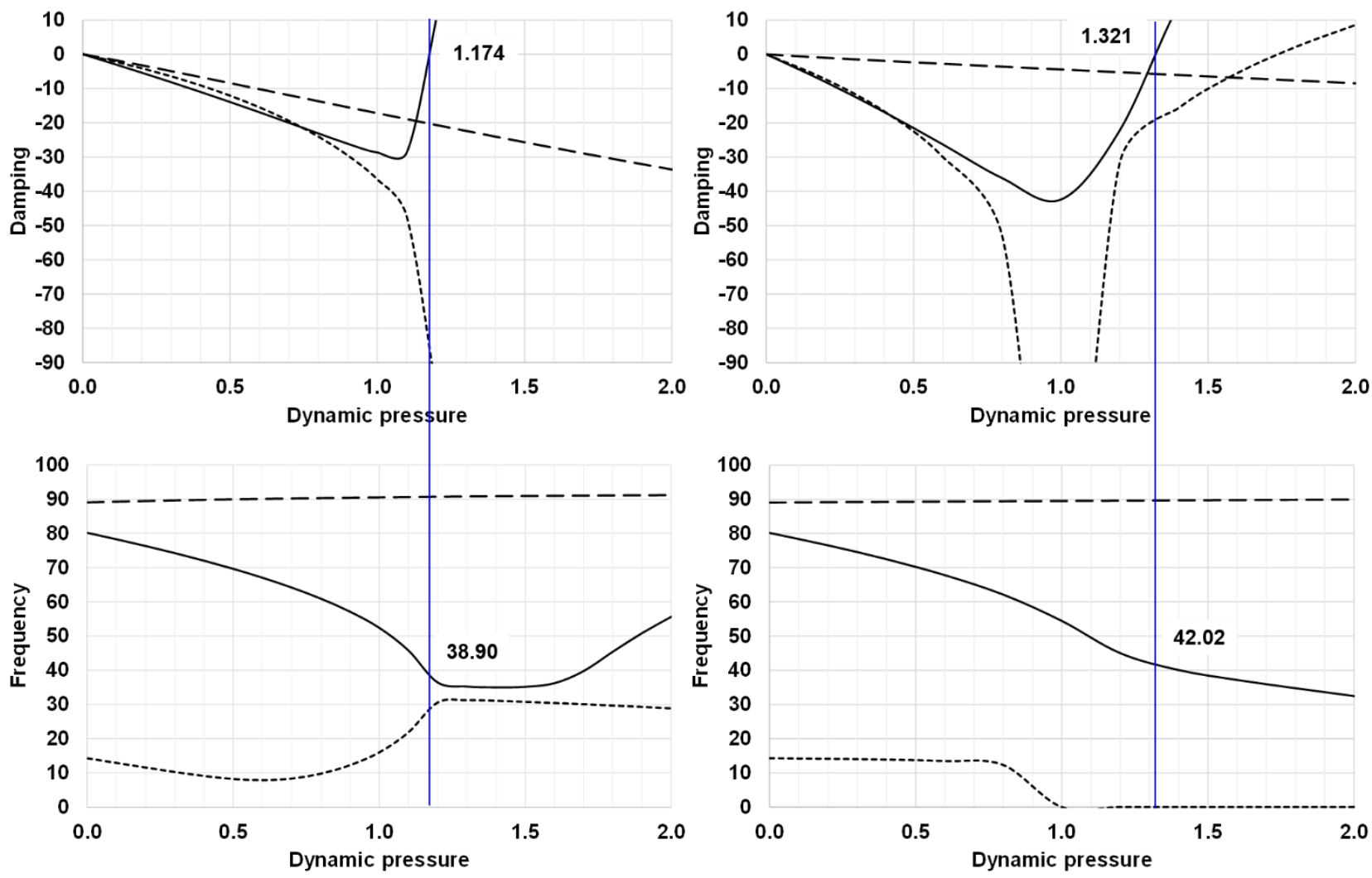

(a) SOCIT $q_{D}=1.00 \mathrm{psi}$

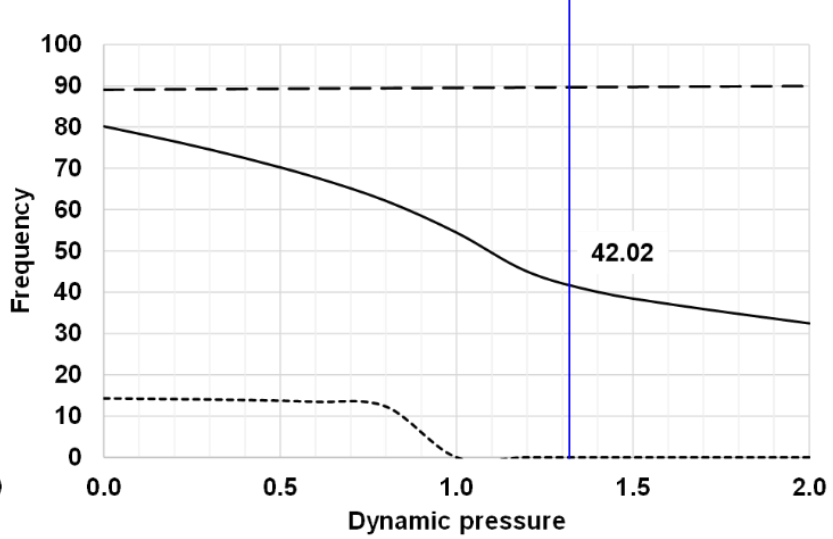

(b) n4sid $q_{D}=1.00 \mathrm{psi}$

Figure 8. $\left(q_{D}-\mathrm{g}\right)$ and $\left(q_{D}-f\right)$ plots for initial $q_{D}=1.0 \mathrm{psi}$. 

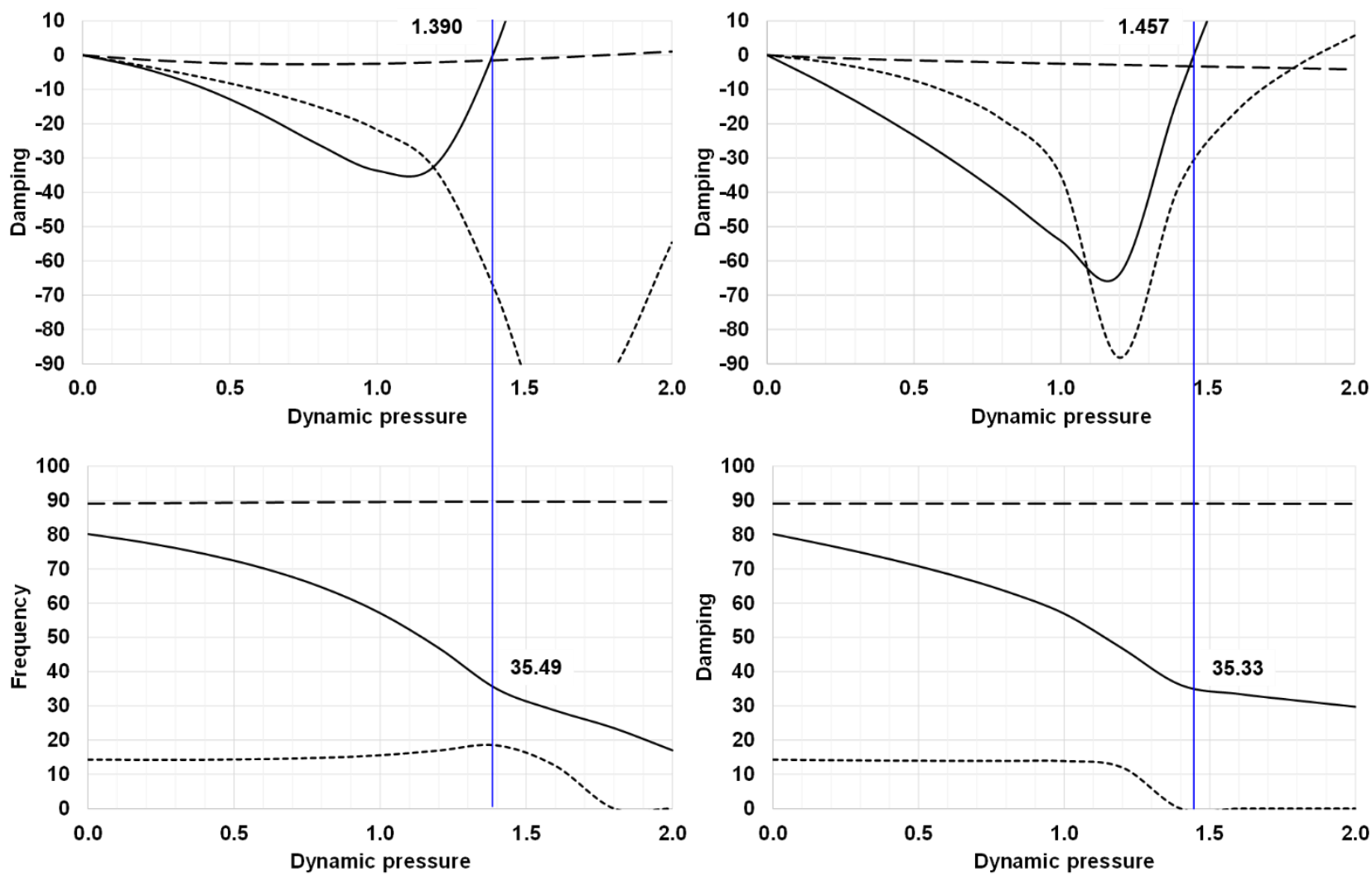

(a) SOCIT $q_{D}=1.20 \mathrm{psi}$

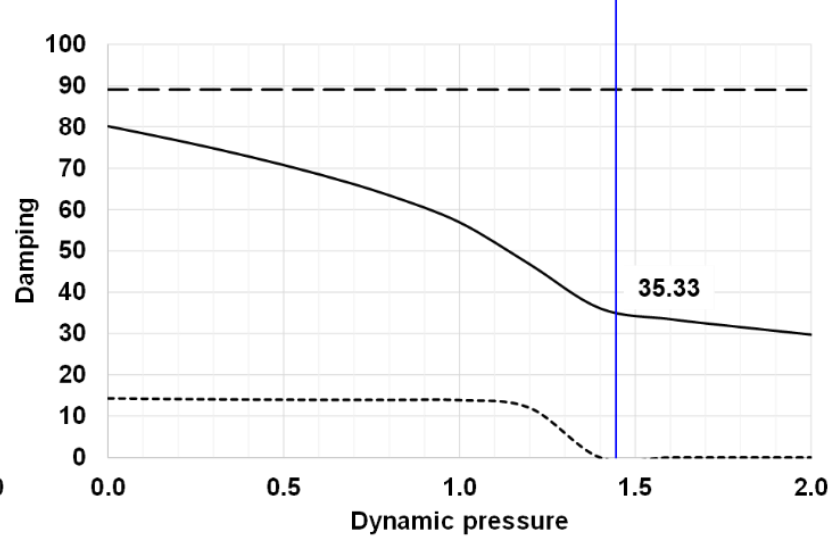

(b) n4sid $q_{D}=1.30 \mathrm{psi}$

Figure 9. $\left(q_{D}-\mathrm{g}\right)$ and $\left(q_{D}-\mathrm{f}\right)$ plots for $\operatorname{SOCIT}\left(q_{\mathrm{D}}=1.20 \mathrm{psi}\right)$ and $\mathrm{n} 4 \operatorname{sid}\left(\mathrm{q}_{\mathrm{D}}=1.30 \mathrm{psi}\right)$. 


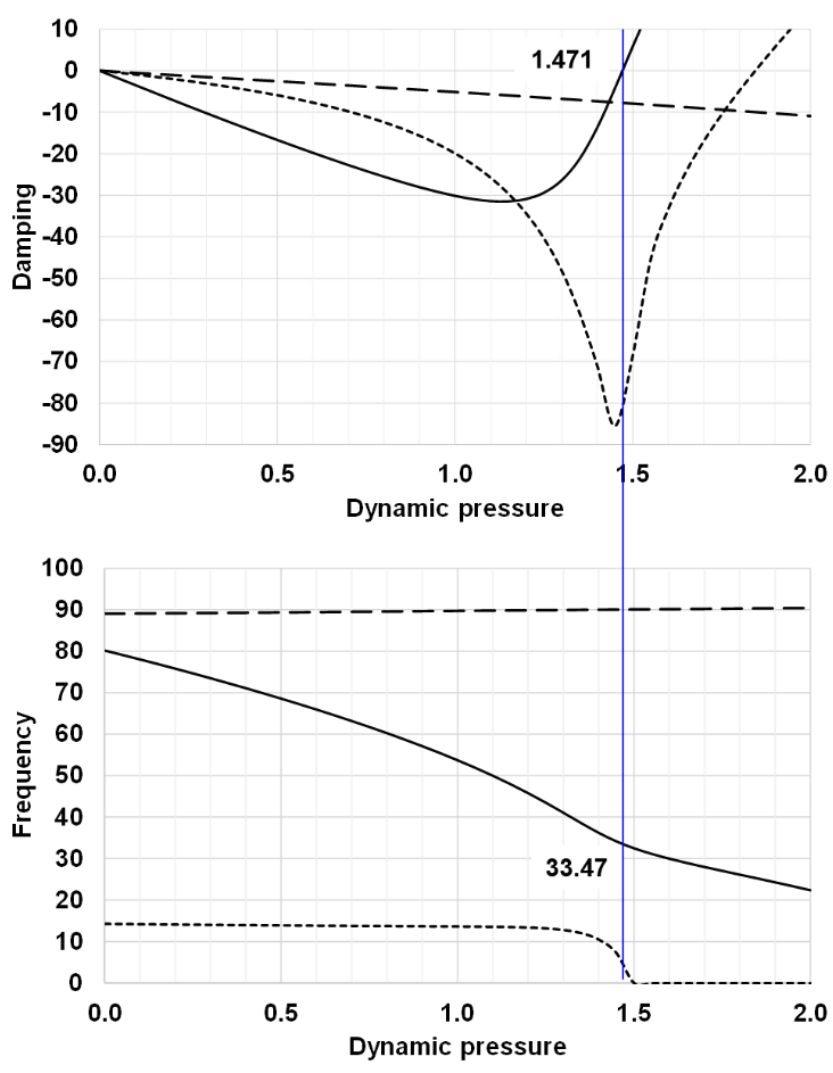

(a) SOCIT $q_{D}=1.40 \mathrm{psi}$
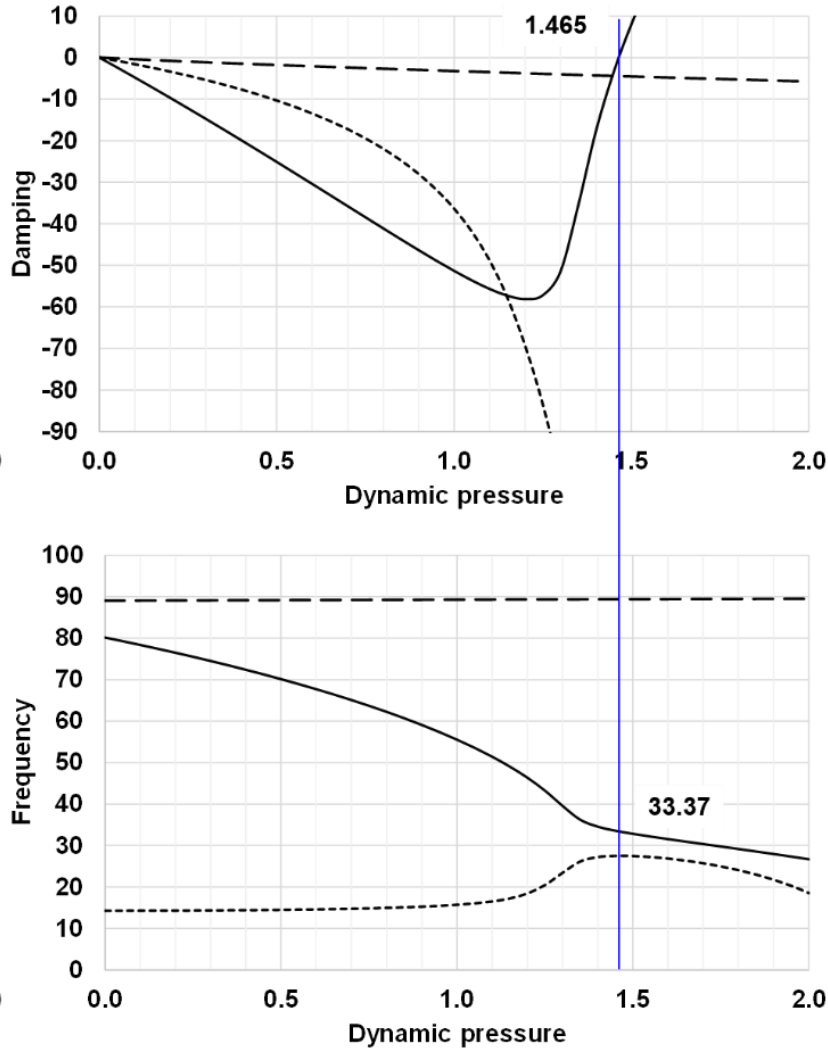

(b) $n 4 s i d q_{D}=1.45 \mathrm{psi}$

Figure 10. $\left(q_{D}-\mathrm{g}\right)$ and $\left(q_{D}-\mathrm{f}\right)$ plots for SOCIT $\left(q_{\mathrm{D}}=1.40 \mathrm{psi}\right)$ and $\mathrm{n} 4 \operatorname{sid}\left(\mathrm{q}_{\mathrm{D}}=1.45 \mathrm{psi}\right)$. 


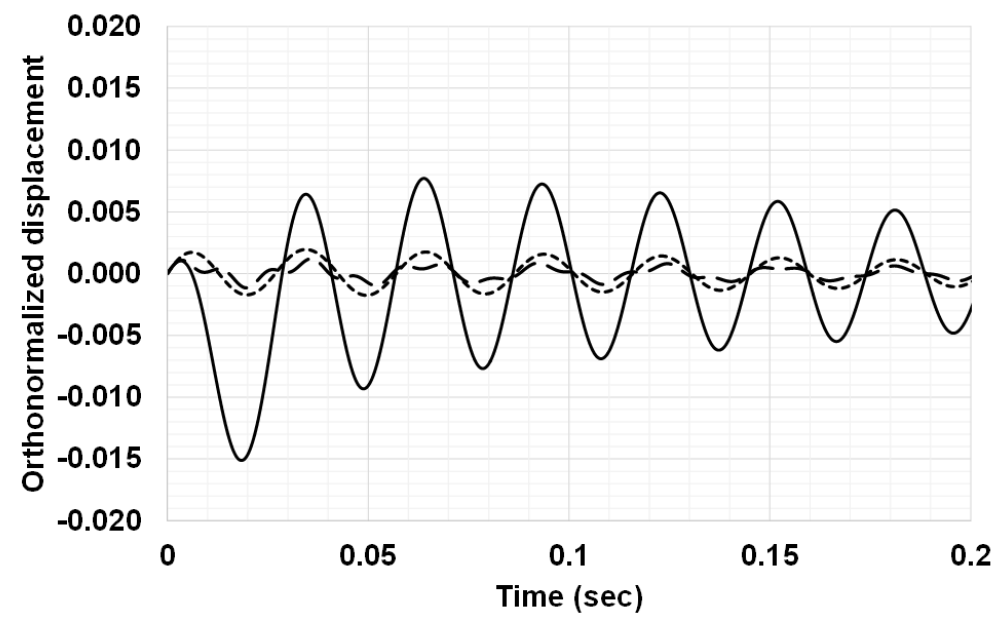

(a) $q_{D}=1.45 \mathrm{psi}$

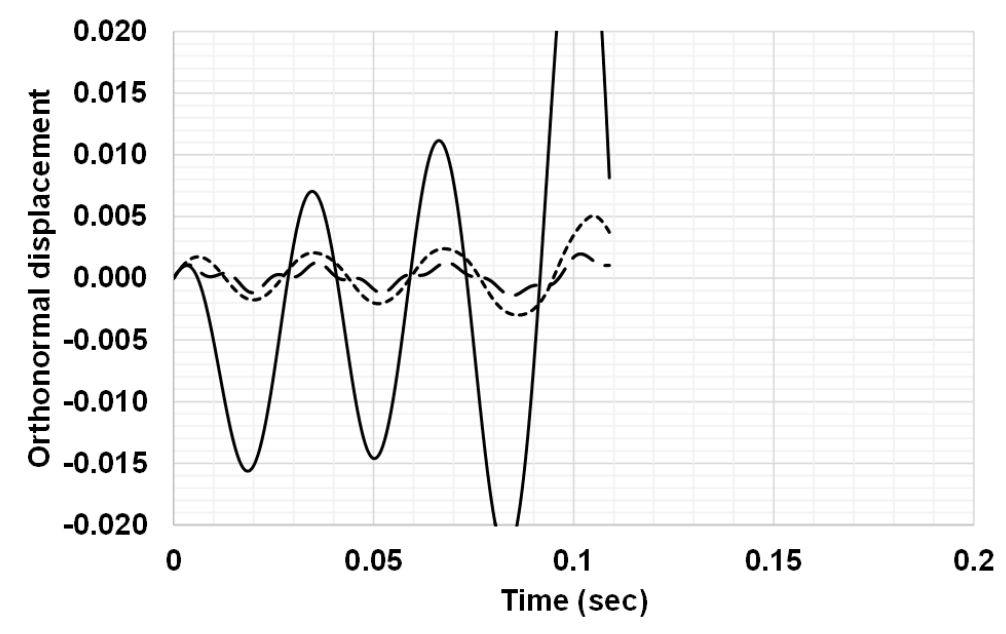

(b) $q_{D}=1.46 \mathrm{psi}$

Figure 11. Time histories of orthonormalized displacement with dynamic pressures of 1.45 and 1.46 psi. 


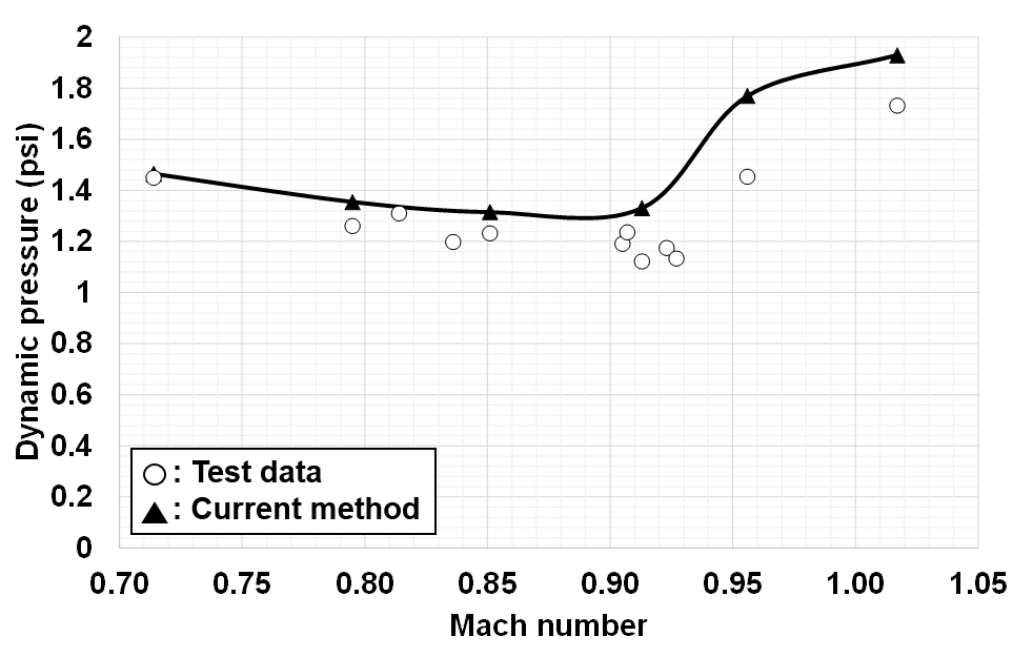

(a) Dynamic pressure

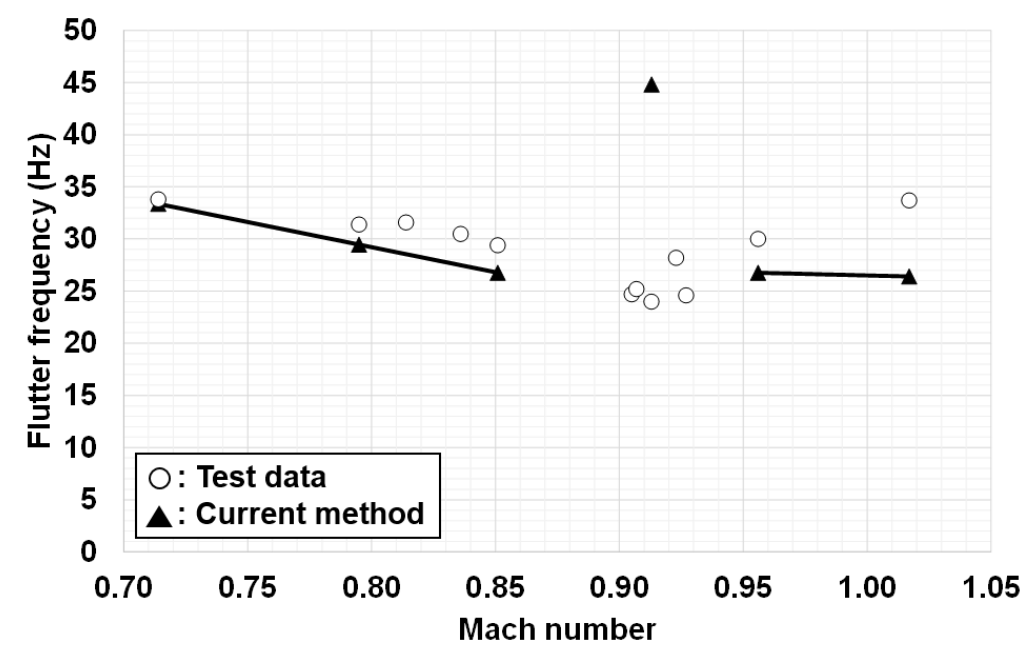

(b) Flutter frequency

Figure 12. Flutter boundary of the cantilevered rectangular wing. 


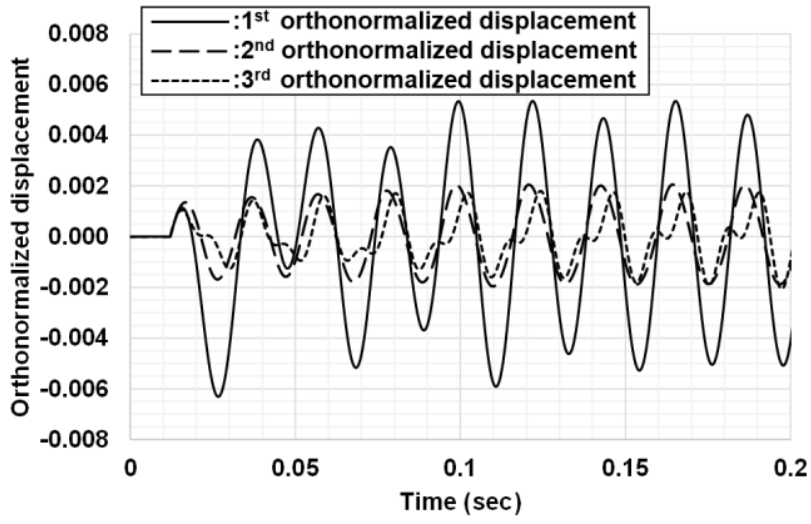

(a) Time histories of orthonormalized displacements

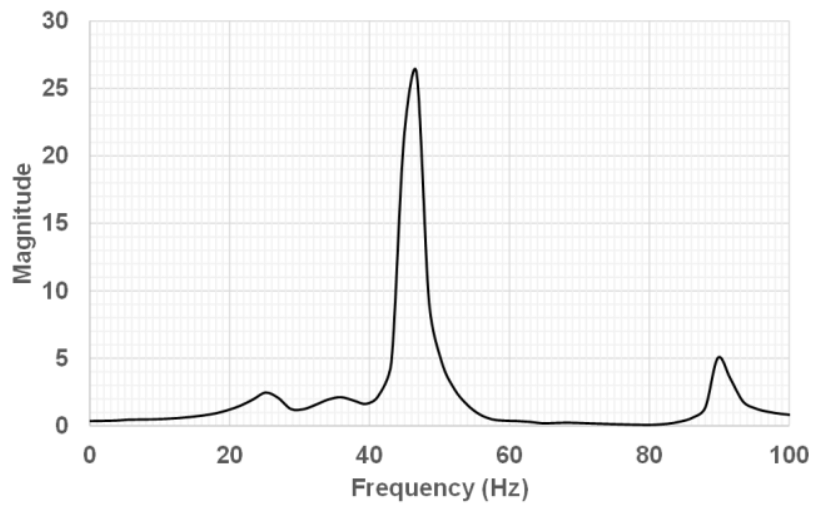

(b) FFT of the first orthonormalized displacement

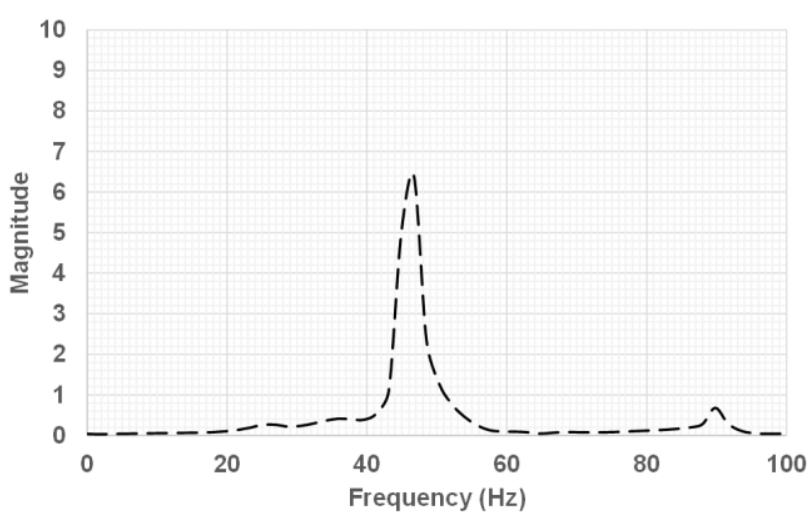

(c) FFT of the second orthonormalized displacement

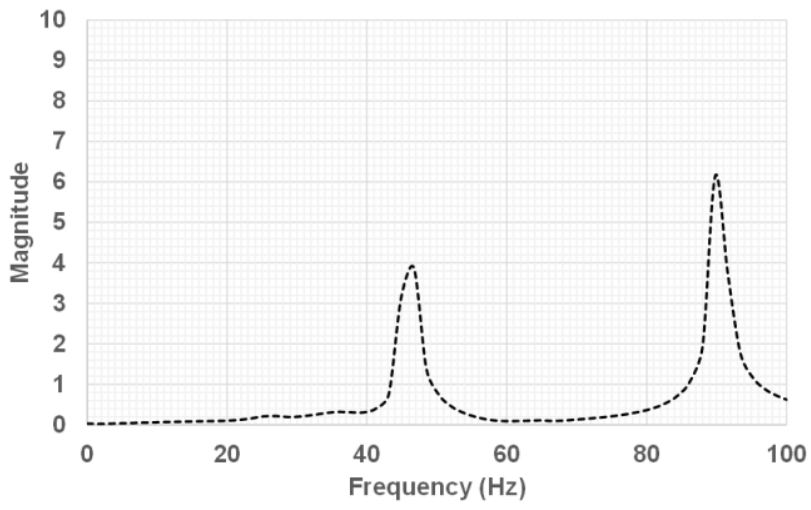

(d) FFT of the third orthonormalized displacement

Figure 13. Time histories and PSDs of the first three orthonormalized displacements. 